\title{
Desempenho de tangerinas precoces enxertadas em citrange Carrizo e citrumelo Swingle
}

\author{
Eduardo Cesar Brugnara ${ }^{1} \&$ Rafael Roveri Sabião ${ }^{1}$
}

\section{RESUMO}

As tangerinas colhidas precocemente geralmente alcançam melhores preços de venda do que as colhidas no pico da colheita. No entanto, informações sobre o desempenho de variedades precoces nas condições edafoclimáticas de Santa Catarina são escassas. Com este estudo, objetivou-se comparar o desenvolvimento vegetativo e a produtividade da Mexirica do Rio IAC, Clemenules, Oronules, Satsumas Okitsu e SCS458 Osvino, enxertadas em citrumelo Swingle e citrange Carrizo, no oeste de Santa Catarina. Para isso as variedades foram avaliadas em um experimento fatorial (copa x porta-enxerto) ao longo de nove anos. As copas diferiram em altura (2,6 a 3,3m), área $\left(7,82\right.$ a $\left.9,96 \mathrm{~m}^{2}\right)$ e volume $\left(13,02\right.$ a $\left.20,84 \mathrm{~m}^{3}\right)$ de projeção do dossel, circunferência do tronco (33,05 a 39,5cm), número (700 a 3350) e massa (98,6 a 405,3kg) acumulada de frutos produzidos por planta, por área e por volume do dossel e massa média dos frutos $(81,6$ a 148,8g). Por outro lado, houve efeito dos porta-enxertos apenas na massa média dos frutos, com interação copa/ porta-enxerto, e na circunferência do tronco das copas. Concluiu-se que as variedades copa mais produtivas são a Mexirica do Rio IAC, Clemenules e Satsuma Okitsu, enquanto Oronules produz poucos e pequenos frutos.

Termos para indexação: Citrus unshiu; Citrus clementina; Citrus deliciosa; porta-enxertos.

\section{Performance of early mandarins grafted on Carrizo citrange and Swingle citrumelo}

\section{SUMMARY}

Early harvested mandarins generally achieve better selling prices than those in harvest season peak. However, information about performance of early harvest varieties in Santa Catarina's edaphoclimatic conditions is scarce. In this study, the objective was to compare the vegetative development and yield of the scion varieties Mexirica do Rio IAC, Clemenules, Oronules, Satsuma Okitsu and SCS458 Osvino, grafted on Swingle citrumelo and Carrizo citrange, in western Santa Catarina state. For this, the varieties were evaluated in a factorial experiment (scion x rootstock) along nine years. The scions differed in height ( 2.6 to $3.3 \mathrm{~m})$, canopy projection area $\left(7.82\right.$ to $\left.9.96 \mathrm{~m}^{2}\right)$ and volume (13.02 to $\left.20.84 \mathrm{~m}^{3}\right)$, trunk circumference $(33.05$ to $39,5 \mathrm{~cm})$, cumulated number ( 700 to 3350 ) and mass (98.6 to $405.3 \mathrm{~kg}$ ) of fruits produced per plant, by canopy projection area and volume and in average fruit mass (81.6 to $148.8 \mathrm{~g}$ ). On the other hand, there was effect of rootstocks

\footnotetext{
${ }^{1}$ Centro de Pesquisa para Agricultura Familiar, Empresa de Pesquisa Agropecuária e Extensão Rural de Santa Catarina - EPAGRI, Chapecó, SC, Brasil

Autor correspondente: Eduardo Cesar Brugnara, Empresa de Pesquisa Agropecuária e Extensão Rural de Santa Catarina - EPAGRI, Servidão Ferdinando Ricieiri Tusset, s/n, CEP 89803-904, Chapecó, SC, Brasil. E-mail: eduardobrugnara@epagri.sc.gov.br
} 
only on the average fruit mass with scion $\mathrm{x}$ rootstock interaction, and on scion trunk circumference. It was concluded that the most productive scions are Mexirica do Rio IAC, Clemenules and Satsuma Okitsu, while Oronules produces few and small fruits.

Index terms: Citrus unshiu; Citrus clementina; Citrus deliciosa; rootstocks.

\section{INTRODUÇÃO}

Tangerinas são frutas muito apreciadas pelo seu sabor e pela facilidade de consumo, quando comparadas às laranjas, pois são facilmente descascadas e seus gomos possuem aderência leve (Koller \& Schäfer, 2009). Muitas variedades, inclusive, não apresentam sementes. A oferta de tangerinas no Brasil é concentrada nos meses de maio a julho, quando se colhe principalmente a Ponkan (Citrus reticulata Blanco), que é a variedade mais apreciada, cultivada e consumida. A maior oferta na época da safra da Ponkan deprime os preços pagos ao produtor e o preço de venda no varejo no mercado catarinense (Barni et al., 2013).

Para atingir melhores preços de venda e o mercado na entressafra da Ponkan, outras variedades são utilizadas. As mais precoces pertencem à espécie Citrus unshiu Marcow (satsumas), não apresentam sementes e podem ser colhidas a partir de fevereiro. Variedades de Citrus clementina hort. ex. Tan. (clementinas), espécie muito cultivada na Espanha, podem ser colhidas um pouco mais tarde e não apresentam sementes quando cultivadas de forma isolada (auto-incompatíveis). Ainda, variedades de Citrus deliciosa Ten. (Mexirica do Rio IAC e Caí) podem ser colhidas antes da Ponkan, mas não tão cedo quanto às espécies supracitadas (Koller \& Soprano, 2013; Brugnara, 2019).

O período de colheita é um fator determinante na escolha da variedade copa. Encontra-se na literatura informações sobre época de colheita e qualidade de frutos nas condições de Santa Catarina que permitem escalonar a colheita de tangerinas, puras ou híbridas, de fevereiro a outubro (Koller \& Soprano, 2013). Porém, há poucas informações sobre o desempenho de variedades de tangerinas no Estado de Santa Catarina e mesmo nas regiões de clima subtropical do Brasil no que concerne à produção e crescimento. A produção das quatro safras iniciais das variedades Mexirica do Rio IAC, Clemenules, Oronules, Satsuma Okitsu e SCS458 Osvino no oeste de Santa Catarina foi avaliada nos porta-enxertos citrumelo Swingle e citrange Carrizo, com superioridade da Mexirica do Rio IAC e do porta-enxerto Swingle (Brugnara, 2017). Porém as plantas de citros apresentam crescimento das copas e da produção por planta além do sexto ano, o que justifica uma nova avaliação com dados de tempo mais longo.
As variedades de porta-enxerto citrumelo Swingle e citrange Carrizo, híbridos de Poncirus trifoliata Raf., possuem resistência a doenças de solo como a gomose e também a geadas, características importantes para serem indicados em substituição ao limão Cravo nas condições catarinenses (Koller \& Soprano, 2013).

O objetivo deste estudo foi comparar o desenvolvimento vegetativo e a produtividade de variedades de tangerinas de maturação precoce, enxertadas sobre os porta-enxertos citrumelo Swingle e citrange Carrizo na região Oeste do estado de Santa Catarina, Brasil.

\section{MATERIAL E MÉTODOS}

O experimento foi instalado em Águas Frias/SC (26 $6^{\circ} 52^{\prime} 54.6^{\prime}$ 'S, 52 51'37’O). O local tem altitude de 378m, clima Cfa (Köppen), média das temperaturas mínimas do ar entre 13 e $13,9^{\circ} \mathrm{C}$, média das temperaturas máximas anuais entre 26 e $26,9^{\circ} \mathrm{C}$ e precipitação pluvial anual média entre 1.700 e $1.899 \mathrm{~mm}$, sem estação seca definida, com probabilidade de atendimento hídrico do mês mais seco (janeiro) de 50 a $60 \%$ (Pandolfo etal., 2002). O solo é um Cambissolo Háplico eutrófico (Potter etal., 2004), cultivado anteriormente com cultivos anuais, tendo apresentado $31 \%$ argila, 2,9\% de matéria orgânica, $\mathrm{pH} 5,65$, índice SMP de 5,7, teor de 6,1 $\mathrm{mg} \mathrm{dm}^{-3} \mathrm{de} P$ e $245 \mathrm{mg}$ $\mathrm{dm}^{-3}$ de K, 7,7 $\mathrm{cmol}_{\mathrm{c}} \mathrm{dm}^{-3}$ de $\mathrm{Ca}, 1,7 \mathrm{cmol}_{\mathrm{c}} \mathrm{dm}^{-3} \mathrm{de} \mathrm{Mg}$. $\mathrm{O}$ manejo, inicialmente, seguiu as diretrizes para produção orgânica. O solo foi previamente corrigido conforme recomendações para a cultura dos citros (Sociedade Brasileira de Ciência do Solo, 2004) com adição de fosfato natural e calcário dolomítico seguido por incorporação com arado de discos em área total. O plantio foi realizado em novembro de 2010, a partir de material de propagação proveniente do banco de germoplasma da Estação Experimental de Itajaí (Epagri), em espaçamento de 7 x $3 \mathrm{~m}$.

O delineamento experimental foi em blocos casualizados, com quatro repetições, em parcelas subdivididas. Nas parcelas principais foram distribuídas as variedades copa: satsuma SCS458 Osvino (C. unshiu), Satsuma Okitsu (C. unshiu), Clemenules (C. clementina), Oronules ( $C$. clementina) e Mexirica do Rio IAC ( $C$. deliciosa); as variedades de porta-enxerto citrumelo Swingle (Citrus paradisi Mac. $\mathrm{x}$ Poncirus trifoliata Raf.) e citrange Carrizo (Citrus 
sinensis (L.) Osb.x Poncirus trifoliata Raf.) constituíram as subparcelas, de três plantas.

Foram realizadas fertilizações suplementares com adubos orgânicos em dose ajustada para suprir a necessidade de nitrogênio (Sociedade..., 2004). Para manejo de doenças foram utilizados sais de cobre e para pragas utilizou-se óleo mineral e óleo de nim (Azadirachta indica A. Juss). A vegetação espontânea foi manejada com capinas e roçadas até o quarto ano, quando se passou à utilização de manejo químico de plantas espontâneas devido à presença de grama Cynodon sp.

As plantas foram podadas anualmente para abertura da copa e quando a carga de frutos era excessiva foi realizado raleio manual. $\mathrm{Na}$ Clemenules, quando havia mais de dois frutos por ramo, retirou-se um; na Satsuma Okitsu e na satuma SCS458 Osvino foram removidos frutos agrupados (pencas); já na Mexirica do Rio IAC foi mantido no máximo um fruto por ramo terminal de forma a ficarem espaçados em cerca de $25 \mathrm{~cm}$. As taxas de raleio atingidas ao longo das safras (média) foram: satsuma SCS458 Osvino - 23,7\%, Satsuma Okitsu - 14,8\%, Clemenules - 0,85\%, e Mexirica do Rio IAC - 24,1\%.

Em novembro de cada ano foram determinadas a altura da planta (H, em metros), a área de projeção da copa - APC, o volume da copa (VC) e a circunferência do tronco das plantas (10 cm acima do enxerto). A APC foi calculada pela equação: $\mathrm{APC}=\pi \times((\mathrm{DT}+\mathrm{DL}) / 4)^{2}$, em que DT é o diâmetro da copa transversal à linha de plantio e DL é o diâmetro longitudinal à linha, em metros. $\mathrm{O} \mathrm{VC}$ foi calculado pela fórmula $\mathrm{VC}=(\mathrm{DT}+\mathrm{DL} / 2) * \mathrm{H} * 0,4909(\mathrm{Coder}, 2000)$.

O número (NF) e a massa (MF) de frutos colhidos por planta foram determinados de fevereiro a junho, conforme a época de maturação (Brugnara, 2019), por contagem manual e aferição de massa em balança. Com os dados das sete safras calculou-se e a massa média dos frutos (MF/NF). Em seqüência, os dados anuais de NF, MF, APC e VC foram agrupados do terceiro ao quinto ano (sub-período jovem), do sexto ao nono ano (sub-período adulto) e do terceiro ao nono ano (período total). Para cada sub-período foram determinadas as eficiências produtivas (MF acumulada no sub-período dividida pela APC ou VC médios do sub-períodos). Por fim, um índice de alternância de produtividade foi calculado com base na equação de Hoblyn (apud Smith et al., 2004), utilizando-se a produção por volume de copa de cada ano ao invés da produção por planta, para reduzir a influência do crescimento da planta.

Os pressupostos da análise de variância foram verificados por testes de BoxCox (pacote MASS do aplicativo R for Windows) (Venables \& Ripley, 2002) e elevação dos dados à potência sugerida. Com os dados transformados quando necessário, os efeitos das copas e porta-enxertos foram analisados por análises de variância complementada pelo teste de Tukey $(\alpha=0,05)$ quando o efeito de tratamento, subtratamento ou sua interação foi significativa, utilizando o aplicativo R provido do pacote Agricolae (Mendiburu, 2016; R Core Team, 2018).

\section{RESULTADOS}

O volume de copa, número e massa de frutos colhidos e massa média dos frutos das plantas ao longo dos nove anos de avaliação são apresentados na Figura 1. No nono ano as plantas ainda apresentaram aumento de volume
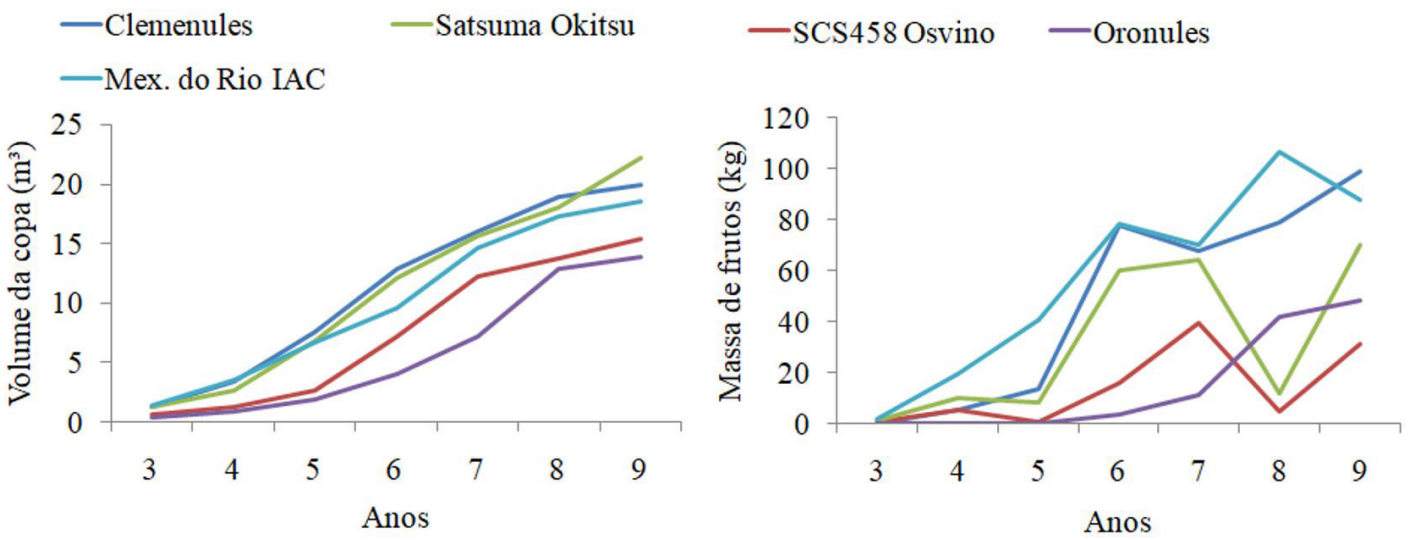

Figura 1. Evolução do volume de copa (a) e da massa de frutos colhidos (b) por plantado $3^{\circ}$ ao $9^{\circ}$ anos de pomar de variedades de tangerinas. Média das plantas enxertadas nos porta-enxertos citrumelo Swingle e citrange Carrizo. Águas Frias, SC, Brasil. Safras 2013 a 2019. 
de copa (Figura 1a), o que indica que provavelmente o tamanho adulto e o pico de produção não foram atingidos. A produção iniciou no terceiro ano e oscilou ao longo das safras (Figura 1b), principalmente nas duas satsumas, Satsuma Okitsu e SCS 458 Osvino, apesar do crescimento contínuo da copa, o que caracteriza a alternância de produção. Destaca-se que a clementina Oronules iniciou a produção de frutos apenas no oitavo ano (sexta safra) e, portanto, as variáveis que envolvem a sua produção de frutos se referem a apenas quatro safras.

$\mathrm{Na}$ análise de variância, a interação entre os fatores experimentais copa e porta-enxerto foi significativa apenas quando considerada a massa média dos frutos no sub-período adulto, e esta variável não foi afetada por nenhum fator no período jovem. Nas demais variáveis resposta houve efeito principal significativo de variedades copa em todas as variáveis, e de porta-enxertos apenas na circunferência do tronco.

A altura das plantas medida no nono ano do pomar foi maior nas copas Satsuma Okitsu, Clemenules e Mexirica do Rio IAC, independente do porta-enxerto (Tabela 1). A satsuma SCS458 Osvino também foi superada pela Satsuma Okitsu. Resultados semelhantes foram observados para área de projeção e volume de copa. Clemenules e Satsuma Okitsu apresentaram APC e VC significativamente maiores. Já a circunferência do tronco foi maior na Clemenules e na Satsuma Okitsu do que na satsuma SCS458 Osvino. A circunferência do tronco das plantas enxertadas em Carrizo foi maior do que nas enxertadas em Swingle.
O número de frutos colhidos no período jovem do pomar foi maior na Mexirica do Rio IAC (Tabela 2), porém, no período adulto e no acumulado total das safras não diferiu significativamente da Clemenules. Essas mesmas diferenças foram observadas na massa de frutos, exceto na comparação de Oronules com Satsuma Okitsu, cuja diferença não foi significativa. A Satsuma Okitsu apresentou desempenho produtivo intermediário, superando a SCS458 Osvino e a Oronules em massa de frutos colhidos nas sete safras. A Oronules, apesar de não ter produzido no período jovem, no período adulto igualou a produtividade da Satsuma Okitsu e SCS458 Osvino. Comparando as duas variedades de C. unshiu, observou-se na SCS 458 Osvino menor massa de frutos colhidos no período adulto e total do que na Satsuma Okitsu.

O índice de alternância de produtividade variou de 0,34 na Mexirica do Rio IAC e Oronules até 0,64 na SCS458 Osvino, cuja diferença foi significativa (Tabela 3 ). Na Clemenules e Satsuma Okitsu o IAP não diferiu das demais.

As plantas de Mexirica do Rio IAC foram as de maior produtividade por área e por volume de copa no pomar jovem, sem diferir significativamente da Clemenules no pomar adulto (Tabela 3). Além disso, as satsumas Satsuma Okitsu e SCS458 Osvino não diferiram em produção por área de projeção e volume da copa. A Oronules não foi incluída na comparação do sub-período jovem por não ter produzido frutos, mas no adulto apresentou eficiência semelhante às satsumas.

A massa média dos frutos não foi afetada pelos tratamentos no sub-período jovem do pomar. No adulto, a análise da interação pelo teste de Tukey dentro de copas informou

Tabela 1. Altura, área de projeção e volume da copa e circunferência do tronco $10 \mathrm{~cm}$ acima da enxertia (CT) de variedades de tangerinas enxertadas em dois porta-enxertos no nono ano do pomar (Águas Frias, SC, Brasil)

\begin{tabular}{|c|c|c|c|c|}
\hline \multirow{2}{*}{ Tratamentos } & Altura & Área & Volume & $\mathrm{CT}$ \\
\hline & $(\mathrm{m})$ & $\left(\mathrm{m}^{2}\right)$ & $\left(m^{3}\right)^{(2)}$ & $(\mathrm{cm})$ \\
\hline \multicolumn{5}{|c|}{ - _ - _ _ - - - Efeito principal de variedade copa $-\ldots \ldots$} \\
\hline Satsuma Okitsu & $3,33 \mathrm{a}$ & $9,96 \mathrm{a}$ & $20,84 \mathrm{a}$ & 39,36 a \\
\hline Mexirica do Rio IAC & $3,11 \mathrm{ab}$ & $8,92 \mathrm{ab}$ & $17,36 \mathrm{~b}$ & $35,57 \mathrm{ab}$ \\
\hline SCS458 Osvino & $2,79 \mathrm{bc}$ & $8,04 \mathrm{bc}$ & $14,38 \mathrm{c}$ & $33,05 \mathrm{~b}$ \\
\hline Oronules & $2,66 \mathrm{c}$ & $7,82 \mathrm{c}$ & $13,02 \mathrm{c}$ & $34,81 \mathrm{ab}$ \\
\hline Clemenules & $3,11 \mathrm{ab}^{(1)}$ & $9,57 \mathrm{a}$ & $18,68 \mathrm{ab}$ & $39,50 \mathrm{a}$ \\
\hline Coef. variação $(\%)$ & 5,9 & 7,1 & 2,5 & 8,2 \\
\hline \multicolumn{5}{|c|}{ - - - - - - Efeito principal de variedade porta-enxerto $-\ldots$} \\
\hline Carrizo & $3,03^{\mathrm{ns}}$ & $8,81^{\mathrm{ns}}$ & $16,9^{\text {ns }}$ & $37,45^{\mathrm{a}}$ \\
\hline Swingle & 3,01 & 8,91 & 16,8 & $35,57 \mathrm{~b}$ \\
\hline C. V. $(\%)$ & 5,3 & 8,1 & 2,5 & 6,8 \\
\hline
\end{tabular}

(1) Médias seguidas pela mesma letra na coluna não diferem significativamente (Teste de Tukey, $p=0,05$ ); ${ }^{(2)}$ variável analisada após transformação (BoxCox) pelo expoente $-0,2 ;{ }^{\text {ns }}=$ não significativo (ANAVA, $p>0,05$ ). 
que a Satsuma Okitsu, a Clemenules e a Mexirica do Rio IAC também não foram afetadas pelos porta-enxertos testados (Tabela 4). Por outro lado, a SCS458 Osvino apresentou, na fase adulta, frutos maiores quando enxertada em Carrizo e a Oronules quando em Swingle. Entre as copas, no sub-período adulto, independente do porta- enxerto, a SCS458 Osvino produziu frutos com maior massa média que as demais copas, porém só superou a Satsuma Okitsu quando enxertadas sobre Carrizo. A Mexirica do Rio IAC e a Clemenules não diferiram em massa média de frutos, enquanto a Oronules apresentou médias inferiores às demais copas.

Tabela 2. Número e massa de frutos colhidos por planta de variedades de tangerinas em Águas Frias, SC, nos sub-períodos jovem (3-5 anos) e adulto (6-9 anos). Os dados são as médias dos porta-enxertos citrumelo Swingle e citrange Carrizo

\begin{tabular}{|c|c|c|c|c|c|c|}
\hline \multirow{3}{*}{ Variedade } & \multicolumn{3}{|c|}{ - - - - - Número frutos - - - - - } & \multicolumn{3}{|c|}{ - - - - - - Massa de frutos - - - - - - } \\
\hline & jovem & adulto & total $^{(1)}$ & jovem & adulto & total $^{(1)}$ \\
\hline & \multicolumn{2}{|c|}{$\left(---n^{0} a^{-1}---\right)$} & $(\mathrm{x} 1.000)$ & \multicolumn{2}{|c|}{$\left(----\mathrm{kg}_{\mathrm{ano}}{ }^{-1}---\right)$} & $(\mathrm{kg})$ \\
\hline Mex. do Rio & $143,49 \mathrm{a}$ & $729,32 \mathrm{a}$ & $3,35 \mathrm{a}$ & $20,74 \mathrm{a}$ & $85,78 \mathrm{a}$ & $405,34 \mathrm{a}$ \\
\hline Clemenules & $40,20 b^{(2)}$ & $665,62 \mathrm{a}$ & $2,78 \mathrm{a}$ & $6,33 \mathrm{~b}$ & $80,64 \mathrm{a}$ & $341,56 \mathrm{a}$ \\
\hline Satsuma Okitsu & $34,28 \mathrm{~b}$ & $373,04 \mathrm{~b}$ & $1,59 \mathrm{~b}$ & $6,27 \mathrm{~b}$ & $51,43 \mathrm{~b}$ & $224,54 \mathrm{~b}$ \\
\hline Oronules & - & $310,68 \mathrm{bc}$ & $1,24 \mathrm{bc}$ & - & $26,27 \mathrm{bc}$ & $105,06 \mathrm{c}$ \\
\hline SCS458 Osvino & $14,83 \mathrm{~b}$ & $163,09 \mathrm{c}$ & $0,70 \mathrm{c}$ & $2,31 \mathrm{~b}$ & $22,93 \mathrm{c}$ & $98,64 \mathrm{c}$ \\
\hline C. V. $(\%)$ & 31,9 & 29,7 & 27,5 & 30,8 & 30,2 & 27,8 \\
\hline
\end{tabular}

(1) Total acumulado nas sete safras; (2) Médias seguidas pela mesma letra na coluna não diferem estatisticamente (Teste de Tukey, $\alpha=0,05$ ).

Tabela 3. Índice de alternância de produção (IAP) $)^{(1)}$ e eficiência produtiva média por área (EPA) e volume da copa (EPV) de variedades de tangerinas em Águas Frias, SC, nos sub-períodos jovem (3-5 anos) e adulto (6-9 anos). Média dos porta-enxertos citrumelo Swingle e citrange Carrizo

\begin{tabular}{|c|c|c|c|c|c|}
\hline \multirow{3}{*}{ Variedade } & \multirow{3}{*}{$\operatorname{IAP}^{(1)}$} & \multicolumn{2}{|c|}{ EPA } & \multicolumn{2}{|c|}{ EPV } \\
\hline & & jovem & adulto & jovem & adulto \\
\hline & & \multicolumn{2}{|c|}{$\left(\mathrm{kg} \mathrm{m}^{-2}\right)$} & \multicolumn{2}{|c|}{$\left(\mathrm{kg} \mathrm{m}^{-3}\right)$} \\
\hline Mexirica do Rio IAC & $0,34 b^{(3)}$ & $6,40 \mathrm{a}$ & $11,79 \mathrm{a}$ & $4,34 \mathrm{a}$ & $6,10 \mathrm{a}$ \\
\hline Clemenules & $0,47 \mathrm{ab}$ & $1,73 \mathrm{~b}$ & $9,51 \mathrm{a}$ & $1,10 \mathrm{~b}$ & $4,89 \mathrm{ab}$ \\
\hline Satsuma Okitsu & $0,53 \mathrm{a}$ & $2,46 b$ & $6,25 \mathrm{~b}$ & $1,94 \mathrm{~b}$ & $3,20 \mathrm{bc}$ \\
\hline Oronules $^{(2)}$ & $0,34 \mathrm{~b}$ & - & $3,91 \mathrm{~b}$ & - & $2,27 \mathrm{c}$ \\
\hline SCS458 Osvino & $0,64 \mathrm{a}$ & $1,75 \mathrm{~b}$ & $3,25 \mathrm{~b}$ & $1,80 \mathrm{~b}$ & $1,86 \mathrm{c}$ \\
\hline C.V. $(\%)$ & 25,7 & 27,7 & 29,7 & 29,8 & 31,7 \\
\hline
\end{tabular}

(1) Calculado com a EPV; (2) Só produziu no sub-período adulto; ${ }^{(3)}$ Médias seguidas pela mesma letra na coluna não diferem estatisticamente (Teste de Tukey, $\alpha=0,05$ ).

Tabela 4. Massa média dos frutos ${ }^{(1)}$ produzidos por cinco variedades de tangerinas enxertadas em dois porta-enxertos do sexto ao nono ano do pomar em Águas Frias, SC

\begin{tabular}{lcc}
\hline \multicolumn{1}{c}{ Variedade copa } & Citrange Carrizo & Citrumelo Swingle \\
\hline SCS458 Osvino & $148,8 \mathrm{aA}^{(2)}$ & $135,8 \mathrm{aB}$ \\
Satsuma Okitsu & $134,5 \mathrm{bA}$ & $139,12 \mathrm{aA}$ \\
Clemenules & $121,59 \mathrm{cA}$ & $121,13 \mathrm{bA}$ \\
Mexirica do Rio IAC & $119,3 \mathrm{cA}$ & $116,54 \mathrm{bA}$ \\
Oronules & $81,6 \mathrm{~dB}$ & $88,82 \mathrm{cA}$ \\
\hline
\end{tabular}

(1) Análise de variância realizada com a variável transformada (BoxCox) pelo expoente $-0,1$; ${ }^{(2)}$ Médias seguidas pela mesma letra minúscula na coluna e maiúscula na linha não diferem estatisticamente (Teste de Tukey, $\alpha=0,05$ ). C.V. das parcelas: 5,3\%; C.V. das subparcelas: $4 \%$. 


\section{DISCUSSÃO}

O presente trabalho e o de Brugnara (2017) são os primeiros de cunho científico a serem publicados com ênfase no desempenho agronômico de variedades de tangerinas e seus porta-enxertos no estado de Santa Catarina. Mesmo no âmbito brasileiro são poucas as informações que permitem comparar o desempenho agronômico das variedades copa avaliadas no presente trabalho.

A satsuma SCS458 Osvino foi uma das variedades de menor crescimento no presente experimento (Tabela 1). Brugnara et al. (2012) reportaram altura média de 2,7 $\mathrm{m}$ aos 15 anos, inferior ao observado em outras variedades. Stuchi et al. (2019) observaram variabilidade de tamanho de plantas entre dez variedades de satsumas, mas nenhuma menor que a Satsuma Okitsu. Portanto, a SCS458 Osvino pode ser considerada uma variedade de tangerina de vigor moderado, menor que Satsuma Okitsu, ao menos quando enxertada em Swingle e Carrizo. A Oronules apresentou copa com tamanho menor que três das quatro variedades desta avaliação (Tabela 1), que pode ser justificado pela baixa expansão da copa nos primeiros anos (Figura 1a). Aznar (1999) descreve a Oronules como variedade de entrenós curtos, a Clemenules como planta de folhagem densa e as satsumas como plantas com brotos vigorosos sem ramificações laterais, que resulta em copa com ramos esparsos. Assim, copas de mesmo volume podem conter quantidades diferentes de folhas e gemas bem como capacidade produtiva. A tese é reforçada pelo menor volume, altura e área de copa associadas à circunferência do tronco sem diferença (Tabela 1).

A maior circunferência do tronco das plantas enxertadas em Carrizo já foi observada com Okitsu em Paranavaí, Paraná (Tazima et al., 2014), mas naquele caso as copas também eram maiores em Carrizo. É possível que as podas realizadas no presente experimento tenham uniformizado o tamanho das copas, mas não do tronco. Por outro lado, em Londrina, Paraná, as plantas de Okitsu cresceram mais quando enxertadas em Swingle que em Carrizo (Tazima et al., 2013), e em Bebedouro, São Paulo, não diferiram em volume de copa (Cantuarias-Avilés et al., 2010).

Na Espanha, a Clemenules enxertada em Swingle apresentou menor volume de copa do que em Carrizo (Arenas et al., 2011). As diferenças de resposta entre locais se devem aos diferentes climas e solos que limitam ou potencializam a expressão do genótipo do porta-enxerto, evidenciando a interação genótipo $\mathrm{x}$ ambiente. Por isso são necessários estudos regionalizados para a seleção de novas variedades.

Os porta-enxertos não influenciaram significativamente a produção de frutos e a eficiência produtiva. Figueiredo et al. (1979) também não observaram efeito significativo de dez porta-enxertos no crescimento, produção e eficiência produtiva da Mexerica do Rio, por causa dos altos coeficientes de variação provocados pela alternância de produção. Cantuarias-Avilés et al. (2010) também não observaram diferenças significativas com dois porta-enxertos na produção de frutos, alternância de produção e eficiência produtiva de Okitsu. Porém, Tazima et al. (2014) observaram maior produtividade acumulada de Okitsu sobre Carrizo, em Paranavaí, Paraná, enquanto Tazima et al. (2013) observaram maior produtividade de Okitsu sobre Swingle em Londrina, Paraná, indicando que os mesmos materiais apresentam variação nos diferentes locais avaliados.

Mesmo nos anos de maior carga (6, 7 e 9$)$ a produção da Satsuma Okitsu foi de 59 a $70 \mathrm{~kg}$ por planta, média dos dois porta-enxertos, inferior aos observado no Paraná (acima de $100 \mathrm{~kg}_{\text {planta }}{ }^{-1}$ nos melhores anos, em plantas com até 11 anos, diferindo em manejo), assim como a eficiência produtiva (Tazima et al., 2013; 2014). Já em Bebedouro, SP, Stuchi et al. (2019) observaram produção de $26,4 \mathrm{~kg}$ por planta de satsuma Okitsu enxertada em Swingle, semelhante ao mensurado em Águas Frias no período equivalente: $26,15 \mathrm{~kg}$ por planta (dados não apresentados). A eficiência produtiva em Bebedouro foi de $4,51 \mathrm{~kg} \mathrm{~m}^{-3}$, maior que a observada neste trabalho, que alcançou 3,2 $\mathrm{kg} \mathrm{m}^{-3}$ no sub-período adulto. As diferenças de desempenho entre locais se devem a diferenças no manejo e nas condições edafoclimáticas. Dados obtidos no leste de Santa Catarina apontam que a satsuma SCS458 Osvino produz $6,68 \mathrm{~kg}$ de frutos por $\mathrm{m}^{3}$ de copa (Maro et al., 2018), maior do que o observado neste experimento. Porém, não se tem informações das condições de manejo, porta-enxerto e ambiente de cultivo.

As satsumas foram menos eficientes em produção por unidade de volume e área de projeção da copa que a Mexirica do Rio IAC. A variedade Mexerica do Rio enxertada sobre Carrizo foi avaliada no estado de São Paulo (Figueiredo et al., 1979), obtendo-se, em plantas adultas, produção média de $7,1 \mathrm{~kg}$ de frutos por $\mathrm{m}^{3}$, ainda maior que a média observada no presente experimento $\left(6,1 \mathrm{~kg}\right.$ por $\left.\mathrm{m}^{3}\right)$. As copas mais densas da mexerica (Schwarz, 2009), quando comparadas às satsumas (Aznar, 1999), podem ter resultado em maior número de flores por volume de copa, potencializando a eficiência produtiva. 
Por outro lado, a frutificação efetiva pode ter sido menor nas satsumas em função da ausência de sementes nos frutos devida a esterilidade masculina e feminina severa (Oliveira \& Scivittaro, 2011; Maro et al., 2018); já que as clementinas são auto-incompatíveis mas produzem sementes quando polinizadas por outras variedades compatíveis (Azevedo et al., 2013) e a Mexirica do Rio IAC é autofértil. Então a frutificação efetiva das últimas é facilitada pela presença de embriões nos frutinhos, os quais facilitam a fixação do fruto pela produção de fitorreguladores.

A Mexirica do Rio IAC apresentou menor alternância de produtividade ao longo dos anos do que a SCS458 Osvino, mesmo tendo produzido mais frutos por metro cúbico de copa e tendo sofrido taxa de raleio semelhante. Isso indica que os critérios de raleio utilizados podem não ser adequados à SCS 458 Osvino. Por outro lado, as satsumas possuem ramificação esparsa (Aznar, 1999), ao contrário das mexericas (Schwarz, 2009), o que pode significar que a última pode suportar maior carga de frutos por unidade de volume de copa.

As satsumas produziram frutos com maior massa média, porém não foi suficiente para compensar a menor quantidade de frutos fixados, resultando em menor eficiência produtiva. Frutos de Clemenules sem sementes apresentam massa média de $50 \mathrm{~g}$ e diâmetro de $4,5 \mathrm{~cm}$ (Azevedo et al., 2013). Então os frutos de Clemenules de Águas Frias provavelmente apresentavam algumas sementes (não avaliado neste trabalho), pois apresentaram massa média de $121 \mathrm{~g}$. Sua boa produtividade e maturação mais precoce que a Mexirica do Rio IAC (Brugnara, 2019) sugerem que seja cultivada em Santa Catarina, mas o seu cultivo isolado de outras variedades deve ser evitado para não produzir frutos muito pequenos. Os frutos da Oronules, com massa média de até 88,82 g, mesmo com baixa produtividade, teriam diâmetro estimado de menos de $6 \mathrm{~cm}$, tamanho considerado inadequado para o mercado de fruta de mesa de mandarinas (Schwarz et al., 1992).

Apesar da menor produtividade das satsumas neste experimento, elas apresentam algumas vantagens como a produção de frutos maiores e a colheita mais precoce que Clemenules, Mexirica do Rio IAC e a grande maioria das variedades não avaliadas neste trabalho. Seu suco alcança o ratio mínimo para colheita de 39 a 48 dias antes da Mexirica do Rio IAC, e a coloração da casca evolui mais precocemente, especialmente a da SCS458 Osvino (Schwarz, 2009; Brugnara, 2019).

Conclui-se que no ambiente do Oeste de Santa Catarina e sob o manejo aplicado neste experimento, que inclui podas e raleio de frutos, os porta-enxertos citrumelo
Swingle e citrange Carrizo causam poucas diferenças no desempenho agronômico das plantas. As variedades copa mais produtivas são Mexirica do Rio IAC, Clemenules e Satsuma Okitsu, e são as mais indicadas para obtenção de frutos mais cedo do que a safra da Ponkan. A Mexirica do Rio IAC se destaca também pela maior produção na fase jovem do pomar. A Oronules é pouco produtiva, tardia em início de produção e produz frutos pequenos, por isso não deve ser cultivada comercialmente.

\section{AGRADECIMENTOS}

Ao Conselho Nacional de Desenvolvimento Científico e Tecnológico (CNPq), à Fundação de Amparo à Pesquisa e Inovação do Estado de Santa Catarina (FAPESC) e à Financiadora de Estudos e Projetos (FINEP) pelo apoio financeiro. Ao Sr Nilton da Silva por permitir o uso de sua propriedade. Aos ex-colegas Dr. Osvino Leonardo Koller, Dr. Luiz Augusto Ferreira Verona e à colega Dra. Luana Aparecida Castilho Maro pela participação no planejamento e execução do projeto.

\section{REFERÊNCIAS}

Arenas, F. J., Hervalejo, A., Prats, T., Salguero, A., \& Forner-Giner, M. A. (2011). Resultados preliminares del comportamiento de Clemenules injertada sobre varios patrones. Vida Rural, 35, 44-48.

Azevedo, F., Borges, R. S., Fávero, M. A. B., Giorgi Neto, R. O., Schinor, E. H., \& Bastianel, M. A. (2013). Polinização cruzada determina a formação de sementes em frutos de clementina Nules. Pesquisa Agropecuária Tropical, 43(1), 88-92.

Aznar, J. S. (1999). Reconocimiento de variedades de cítricos en campo (Sèrie Divulgación Tècnica, n. 43, 187 pp.). Valencia: ESP, Generalitat Valenciana, Conselleria de Agricultura, Pesca y Alimentación.

Barni, E. J., Koller, O. L., \& Silva, M. C. S. (2013). Mercado catarinense de citros. In O. L. Koller (Org.), Citricultura catarinense (pp. 17-40). Florianópolis: Epagri.

Brugnara, E. C., Verona, L. A. F., \& Koller, O. L. (2012). Crescimento de quatro variedades de tangerineiras enxertadas em quatro porta-enxertos em Chapecó, SC. In Anais do XXII Congresso Brasileiro de FruticulturaI [1 CD-ROM]. Bento Gonçalves, RS: SBF. 
Brugnara, E. C. (2017). Desempenho de tangerineiras jovens, de maturação precoce, enxertadas em Swingle ou Carrizo no Oeste de Santa Catarina, Brasil. Agropecuária Catarinense, 30(2), 69-73.

Brugnara EC (2019). Maturation of early-ripening mandarin as affected by scion and rootstock cultivars in western Santa Catarina, Brazil. Revista Ceres, 66(4), 249-156.

Cantuarias-Avilés, T, Mourão Filho, F. A. A., Stuchi, E. S., Silva, S. R. \& Espinoza-Núñez, E. (2010). Tree performance and fruit yield and quality of Okitsu Satsuma mandarin grafted on 12 rootstocks. Scientia Horticulturae, 123(3), 318-322.

Coder, K. D. (2000). Crown Shape Factors \& Volumes (Tree Biomechanics Series, pp. 1-5). Athens: The University of Georgia.

Figueiredo, J. O., Pompeu Junior, J., Rodriguez, O., \& Caetano, A. A. (1979). Competição de dez porta-enxertos para a mexeriqueira-do-rio (Citrus deliciosa Tenore) (pp. 442-453). Pelotas, RS: Anais do Congresso Brasileiro de Fruticultura.

Koller, O. C., \& Schäfer, G. (2009). Origem da cultura da tangerineira, importância no mundo e no Brasil. In: O. C. Koller (Coord.), Citricultura: cultura de tangerineiras: tecnologia de produção (pp. 13-24). Porto Alegre: Rigel.

Koller, O. L., \& Soprano, E. (2013). Principais cultivares cítricos. In: O. C. Koller (Org.). Citricultura catarinense (pp. 57-120). Florianópolis: Epagri.

Maro, L. A. C., Koller, O. L., Mariguele, K. H., Brugnara, E. C., \& Soprano, E. (2018). 'SCS458 Osvino': novo cultivar de tangerineira precoce com alto potencial produtivo, tolerância ao frio e ausência de sementes. Agropecuária Catarinense, 3(1), 52-55.

Mendiburu, F. (2016). Agricolae: Statistical Procedures for Agricultural Research. R Package version 1.2-1. Recuperado em 31 de agosto de 2016, de http://CRAN.Rproject.org/package=agricolae.

Oliveira, R. P., \& Scivittaro, W. B. (2011). Cultivo de citros sem sementes (378 pp). Pelotas, RS: Embrapa Clima Temperado.

Pandolfo, C., Braga, H. J., Silva Júnior, V. P., Massignan, A. M., Pereira, E. S., Thomé, V. M. R., \& Valci, F. V. (2002). Atlas climatológico do Estado de Santa Catarina. Florianópolis: Epagri.

Potter, R. O., Carvalho, A. P., Flores, C. A., \& Bognola, I. (2004). Solos do Estado de Santa Catarina (729 pp). Rio de Janeiro: Embrapa Solos. Recuperado em 15 de maio de 2017, de http://ainfo.cnptia.embrapa.br/digital/ bitstream/item/88186/1/BPD-46-2004-Santa-Catarina-.pdf

R Core Team (2018). R: A language and environment for statistical computing. Vienna, Austria: R Foundation for Statistical Computing. Recuperado em 15 de maio de 2018, de https://www.R-project.org/.Sociedade Brasileira de Ciência do Solo. Comissão de Química e Fertilidade do Solo. (2004). Manual de adubação e de calagem para os Estados do Rio Grande do Sul e de Santa Catarina (10. ed., 102 pp). Porto Alegre: Sociedade Brasileira de Ciência do Solo.

Schwarz, S. F., Koller, O. C., \& Nienow, A. A. (1992). Intensidades e épocas de raleio manual em tangerineira 'Montenegrina'. Pesquisa Agropecuária Brasileira, 27(8), 1161-1165.

Schwarz, S. F. (2009). Melhoramento genético e variedades. In: O. C. Koller (Coord.), Citricultura: cultura de tangerineiras: tecnologia de produção (pp. 35-48). Porto Alegre: Rigel.

Smith, M. W., Shaw, R. G., Chapman, J. C., Owen-Turner, J., Lee, S., McRae, B. K., Jorgensen, K. R., \& Mungomery, W. V. (2004). Long-term performance of 'Ellendale' mandarin on seven commercial rootstocks in sub-tropical Australia. Scientia Horticulturae, 102(1), 75-89.

Stuchi, E. S., Girardi, E. A., Silva, S. R., Cantuarias-Avilés, T., Parolin, L. G., Reiff, E. T., \& Sempionato, O. R. (2019). Satsuma mandarins grafted onto Swingle citrumelo for early season harvest in subtropical conditions in Brazil. Bragantia, 78(2), 236-243.

Tazima, Z. H., Neves, C. S. V. J., Yada, I. F. U., \& Leite Júnior, R. P. (2013). Performance of 'Okitsu' Satsuma Mandarin on nine rootstocks. Scientia Agrícola, 70(6), 422-427.

Tazima, Z. H., Neves, C. S. V. J., Yada, I. F. U., \& Leite Júnior, R. P. (2014). Performance of 'Okitsu' satsuma mandarin trees on different rootstocks in Northwestern Paraná State. Semina: Ciências Agrárias, 35(5), 2297-2308.

Venables, W. N., \& Ripley, B. D. (2002). Modern Applied Statistics with S (4. ed., 495 pp.). New York: Springer.

Recebido: Outubro 30, 2019 Aceito: Julho 04, 2020

Como citar: Brugnara, E. C. \& Sabião, R. R. (2020). Desempenho de tangerinas precoces enxertadas em citrange Carrizo e citrumelo Swingle. Citrus Research \& Technology, volume, e1058. https://doi.org/10.4322/crt.20619 